\title{
Taxa de geração e composição de resíduos sólidos provenientes de serviço de
}

\section{hotelaria}

\author{
Generation rate and composition of solid waste from hospitality enterprises \\ Tasa de generación y composición de los residuos sólidos generados en servicios hoteleros
}

\section{Resumo}

A expansão do setor hoteleiro resulta em uma também crescente geração de resíduos sólidos provenientes deste setor, sendo considerada um dos impactos mais significativos causados pelos estabelecimentos de hospedagem. Assim, este artigo tem como objetivo analisar a geração de resíduos sólidos na indústria do turismo, mais especificamente no setor hoteleiro. As bases de dados utilizadas para a realização desta revisão foram: Scopus, Scielo, Science direct e Web of science. Como resultado, foram identificados mais de 300 artigos, dos quais 51 se encaixaram nos critérios adotados para análise. A taxa de geração de resíduos sólidos no setor hoteleiro pode variar com inúmeros fatores, como o número de quartos, quantidade de leitos e serviços oferecidos. Deste modo, a geração média per capita de resíduos sólidos apresenta grande variação, de 0,32 a 6,57 kg.hóspede-1.dia-1. Os resíduos sólidos gerados pelos hotéis são compostos basicamente por resíduos orgânicos (57\%) e resíduos secos (32\%). Assim, os resíduos sólidos gerados no setor hoteleiro apresentam grande potencial para reciclagem, já que os resíduos orgânicos podem ser reciclados por meio de compostagem ou biometanização e os secos podem ser reutilizados, reciclados ou aproveitados energeticamente.

Palavras-chave: Gestão de resíduos sólidos; Geração de resíduos sólidos; Hotelaria; Hotéis.

\begin{abstract}
The expansion of the hotel sector results in an increasing generation of solid waste from this sector, being considered one of the most significant impacts caused by hospitality establishments. This article aims to analyze the generation of solid waste in the tourism industry, more specifically in the hotel sector. The databases used to carry out this review were: Scopus, Scielo, Science direct and Web of science. As a result, 300 articles were identified, of which 51 met the criteria adopted for analysis. The rate of solid waste generation in the hotel sector can vary with numerous factors, such as the number of rooms, number of beds and services offered. Thus, the average per capita generation of solid waste varies widely, from 0.32 to $6.57 \mathrm{~kg}$.guest-1.day-1. The solid waste generated by hotels is basically composed of organic waste $(57 \%)$ and dry waste $(32 \%)$. Thus, solid waste generated in the hotel sector has great potential for recycling, since organic waste can be recycled through composting or biomethanization and dry waste can be reused, recycled or used energetically.
\end{abstract}

Keywords: Solid waste managemaent; Solid waste generation Hospitality services; Hotels.

\section{Resumen}

La expansión del sector hotelero se traduce en una creciente generación de residuos sólidos de este sector, siendo considerada uno de los impactos más significativos provocados por los estabelecimientos de hospedaje. Este articulo tuve como objetivo analizar la generación de residuos sólidos en el sector hotelero. Para tanto ha sido utilizada las siguientes bases de datos: Scopus, Scielo, Science direct e Web of Science. Como resultado, se identificaron más de 300 artículos, de los cuales 51 fueron analizados. La tasa de generación de residuos sólidos con origen en lo sector hotelero puede variar con numerosos factores, como el número de habitaciones, cantidad de camas, e servicios ofrecidos. Por lo tanto, la generación media per cápita de residuos sólidos se presenta con una variación de 0,32 a 6,57 kg. huésped1. día-1. Los residuos generados en hoteles son compuestos básicamente por residuos orgánicos (57\%) y residuos secos (32\%). Por fin, los residuos sólidos generados en el sector hotelero tienen un gran potencial de reciclaje, pues pueden reciclarse mediante compostaje o biometanización y los residuos secos pueden ser reutilizarse, reciclarse o aprovechados energéticamente.

Palabras clave: Gestión de residuos sólidos; Generación de residuos sólidos; Hospitalidad; Hoteles. 


\section{Introdução}

No período compreendido entre os anos de 2007 e 2017, a contribuição do setor do turismo com o PIB mundial saltou de US\$ 6 trilhões para US\$ 8,3 trilhões, 3,2\% do PIB global. Este desenvolvimento pode ser explicado por meio da criação de empregos, já que o turismo está relacionado direta ou indiretamente com 313 milhões de empregos em todo o mundo (Wttc, 2018; Wttc, 2017). No Brasil, por exemplo, esta expansão pode ser visada a partir do crescimento de meios de hospedagem existentes no país: o número de meios de hospedagem existentes nas capitais dos estados brasileiros saltou 15\% entre os anos de 2011 e 2016, alcançando a marca de 31 mil, com uma oferta de 1.011.254 unidades habitacionais (quartos, chalés e suítes), e 2 milhões de leitos $17 \%$ e $15 \%$ a mais que em 2015, respectivamente (Ibge, 2017).

O crescimento do setor hoteleiro, se realizado de forma desordenada, poderá representar uma preocupação no tocante à degradação ambiental (Arbulú et al., 2015; Zorpas et al., 2014; Subbiah \& Kannan 2011). Um dos impactos negativos decorrentes do crescimento da indústria hoteleira é a geração de resíduos sólidos (Pirani \& Arafat, 2014). Subbiah e Kannan (2011) destacam o fato de que esse setor consome muitos recursos naturais, como energia e água, aliados a uma grande geração de resíduos sólidos, o que potencializa a importância de uma gestão sustentável destes estabelecimentos.

O gerenciamento inadequado dos resíduos sólidos gerados nos hotéis pode acarretar em problemas para o estabelecimento, como a proliferação de insetos e vetores de doenças, e a emissão de odores, criando um ambiente ruim para hóspedes e funcionários (Appaw-Agbola; Freeman, 2015). No âmbito das regiões turísticas, Muñoz e Navia (2015) reportam que um dos impactos mais relevantes diz respeito à ausência de sistemas de gerenciamento de resíduos sólidos nestas regiões. Ainda segundo os autores, a inexistência deste sistema pode provocar a degradação do meio ambiente devido à disposição incorreta dos resíduos sólidos, como a contaminação das águas superficiais e subterrâneas, emissões de poluentes e proliferação de vetores.

De acordo com Appaw-Agbola e Freeman (2015), os maiores geradores de resíduos sólidos dentro dos hotéis são os setores de cozinha, restaurante e departamento de limpeza. Concordando os autores Guidoni et al. (2018), incluem também os banheiros e os quartos como setores geradores de resíduos sólidos em um hotel. Já Chaabane, Nassour e Nelles (2018) afirmam que jardins e as salas administrativas também contribuem com a geração de resíduos sólidos em hotéis.

Neste contexto é primordial que os resíduos gerados na indústria do turismo, mais especificamente no setor hoteleiro, sejam geridos de forma efetiva. Assim, este artigo tem como objetivo analisar a geração de resíduos sólidos na indústria do turismo, mais especificamente no setor hoteleiro.

\section{Metodologia}

Esta pesquisa trata-se de um estudo qualitativo, sendo caracterizado pela importância da interpretação dos dados por parte do pesquisador (Pereira et al., 2018). A análise da geração de resíduos sólidos no setor hoteleiro foi realizada a partir de uma revisão bibliográfica, definida como sendo aquela desenvolvida a partir de uma base de material já elaborado, constituída principalmente de livros e artigos científicos (Gil, 2002). Foram utilizadas as seguintes bases de dados: Scopus, Science Direct, Scielo e Web of Science. Nestas bases foram pesquisados os seguintes termos: Hospitality Industry; Hotel Industry; e Tourism, todos eles acoplados dos termos Solid Waste Generation ou Solid Waste Management. Foram analisados os artigos publicados no período 2010-2020, e referentes à gestão de resíduos sólidos no setor hoteleiro.

Foram identificados mais de 300 artigos, dos quais 51 foram publicados no período 2010-2020 e tratam diretamente da geração e composição de resíduos sólidos oriundos do setor hoteleiro. Dos 51 artigos estudados, 26 abordam diretamente a geração de resíduos sólidos no setor hoteleiro, avaliando sua quantificação, composição e gerenciamento. Por outro lado, 25 artigos abordam a geração de resíduos em áreas e atividades turísticas, incluindo os hotéis e como a indústria do turismo influencia na geração de resíduos nestas zonas. A Figura 1 ilustra a distribuição espacial dos artigos analisados. 
Figura 1. Distribuição espacial dos artigos analisados.

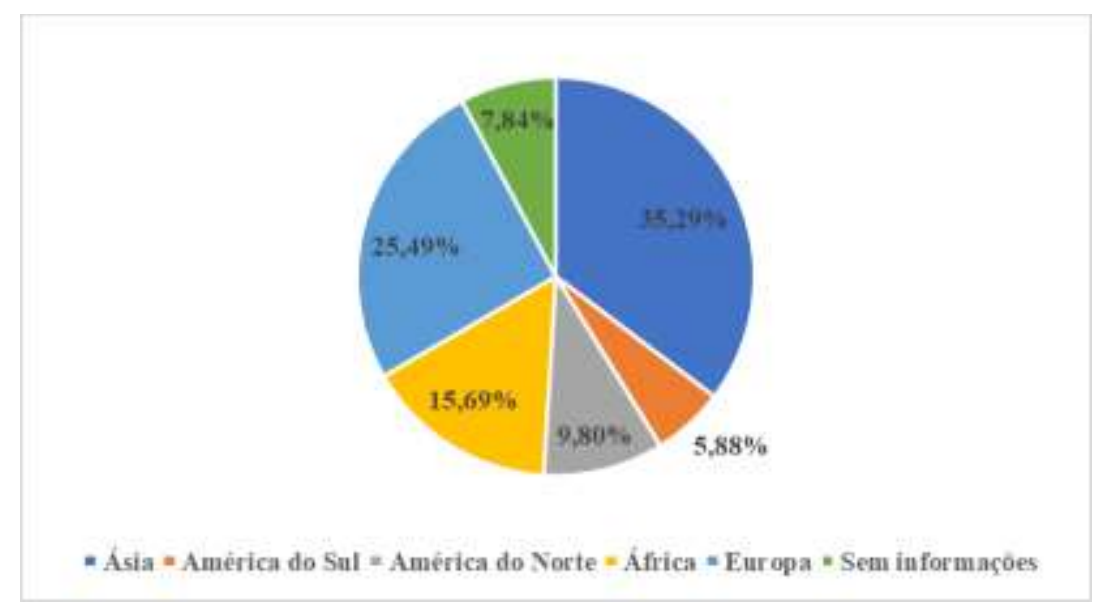

Fonte: Autores (2020).

De acordo com a Figura 1, os artigos analisados estão distribuídos em 28 países e cinco continentes, sendo a Ásia o mais representativo. Salienta-se que dos artigos abordados, apenas quatro foram aplicados ao Brasil, mostrando que a temática ainda é pouco abordada neste país.

\section{Resultados e Discussão}

\subsection{Fatores que influenciam na geração de resíduos sólidos no setor hoteleiro}

A geração de resíduos sólidos no setor hoteleiro é influenciada por vários fatores. Abdulredha et al. (2018) apontam alguns fatores que têm uma influência significativa, como o número de leitos, área do hotel, número de funcionários e os gastos do hotel para com os hóspedes, com o coeficiente de Pearson (PCC) de 0,72, 0,54, 0,61 e 0,62, respectivamente. Os autores ainda afirmam que há uma diferença na geração de resíduos sólidos quanto à classificação dos empreendimentos, sendo os hotéis de quatro estrelas maiores geradores em relação aos hotéis com uma classificação inferior. Corroborando com os autores supracitados, Hoang, Fujiwara e Phu (2017) afirmam que a variação da taxa de geração de resíduos sólidos no setor hoteleiro pode ser explicada em $91 \%$ pelo número de quartos do hotel, sendo os hotéis de grande porte (quatro estrelas) maiores geradores que os hotéis de pequeno porte (hotéis de três e duas estrelas).

Ball e Taleb (2011) reportam que há uma correlação significativa entre o número de quartos, ocupação do hotel e a taxa de geração per capita de resíduos sólidos, com o coeficiente de Pearson (PCC) de 0,52 e 0,45, respectivamente. Concordando com os autores citados anteriormente, Bashir e Goswami (2016) explicam que a geração per capita de resíduos sólidos é mais alta em hotéis classe A, aqueles com 57 quartos e 115 leitos, em relação aos hotéis de classe B, aqueles que possuem, em média, 26 quartos e 52 leitos, e hotéis classe $\mathrm{C}$, aqueles com menos de 23 quartos e 46 leitos.

Phu et al. (2018) demonstram que há uma correlação entre o número de quartos, o valor da diária dos hotéis e a taxa de geração de resíduos sólidos, com o P-Valor menor que 0,05 , sendo a geração per capita influenciada significativamente por estes parâmetros. Os autores ainda determinaram que a taxa de geração de resíduos aumenta em 0,035 kg.hóspede-1.dia-1 para cada quarto adicional com $\mathrm{r}^{2}$ de 0,82 . Um destaque importante aos serviços ofertados pelos hotéis é dado pelos autores, que explicam através do Teste de Kruskal-Wallis que há uma diferença significativa na taxa de geração de resíduos sólidos dos hotéis que não oferecem refeições para os que dispõem deste serviço, como café da manhã e jantar. Também há diferença estatisticamente significativa (P-Valor $<0,05)$ na taxa de geração dos hotéis com jardins e sem jardins.

Assim, de acordo com os autores supracitados a taxa de geração de resíduos sólidos do setor hoteleiro pode variar de acordo com inúmeros fatores, como o número de quartos, número de leitos, área total, número de funcionários, ocupação do 
hotel, classificação do hotel e os serviços oferecidos pelo estabelecimento, de modo que quanto mais completos forem os serviços ofertados, maior será a geração de resíduos sólidos.

\subsection{Taxa de geração per capita de resíduos sólidos do setor hoteleiro}

Analisando uma amostra de 150 hotéis em Querbala, Iraque, Abdulredha et al. (2018) mostram que a taxa de geração per capita de resíduos sólidos varia entre 0,12 e 3,51kg.hóspede-1.dia-1, conforme a classificação dos hotéis, abrangendo aqueles sem classificação e entre uma e quatro estrelas. Em média, esta variação é de 0,83 kg.hóspede-1.dia-1, para hotéis de uma a três estrelas, e 1,22 kg.hóspede-1.dia-1, para hotéis de quatro estrelas. A taxa média de geração per capita para os 150 hotéis foi de 0,89 kg.hóspede-1.dia-1.

Investigando a geração de resíduos sólidos em 24 hotéis cinco estrelas, localizados no Cairo (Egito), Ball e Taleb (2011) determinaram uma taxa de geração per capita dos hotéis analisados variando de 0,4 a 2,8 kg.hóspede-1.dia-1, dependendo das características do hotel, com uma média de 1,5 kg.hóspede-1.dia-1 de resíduos gerados. Em pesquisa realizada em 230 hotéis instalados na cidade de Pahalgan, Índia, Bashir e Goswami (2016) corroboram os autores citados anteriormente e reportaram que hotéis Classe A (57 quartos e 114 leitos) apresentaram uma geração per capita de 2,9 kg.hóspede-1.dia-1, enquanto que hotéis Classe B (26 quartos e 52 leitos) geraram em média 1,9 kg.hóspede-1.dia-1 e hotéis Classe C (23 quartos e 46 leitos) produziram até 1,7 kg.hóspede-1.dia-1 de resíduos. Os autores ainda estimam que as Guest Houses, empreendimentos com menos de 17 quartos geraram cerca de 0,9 kg.hóspede-1.dia-1.

Assim como os autores citados anteriormente, Phu et al. (2018) também destacam a relação entre a geração de resíduos sólidos e o porte do empreendimento. Em estudo envolvendo 120 hotéis localizados em Hoi An, Vietnã, os autores relatam que hotéis de grande porte (80 a 217 quartos) apresentaram uma geração de resíduos sólidos per capita de 6,29 kg.hóspede-1.dia-1, contra 2,69 e 1,34 kg.hóspede-1.dia-1 para hotéis de médio (20 a 80 quartos) e pequeno porte (menos de 20 quartos), respectivamente. Analisando a geração de resíduos sólidos de 45 hotéis com classificação entre Guest Houses e hotéis cinco estrelas e instalados na cidade de Hue, também no Vietnã, Son et al. (2018) mostram resultados semelhantes aos de Phu et al. (2018), com a taxa média de geração de resíduos sólidos variando entre 0,6 kg.hóspede-1.dia-1 para Guest Houses (8 quartos) e 6,57 kg.hóspede-1.dia-1 para hotéis de cinco estrelas (162 quartos). A Tabela 1 apresenta a geração per capita de resíduos sólidos no setor hoteleiro reportada em pesquisas realizadas entre os anos de 2010 e 2020, por diferentes autores de diferentes localidades. 
Research, Society and Development, v. 10, n. 1, e16810111635, 2021

(CC BY 4.0) | ISSN 2525-3409 | DOI: http://dx.doi.org/10.33448/rsd-v10i1.11635

Tabela 1. Geração per capita de resíduos sólidos no setor hoteleiro.

\begin{tabular}{|c|c|c|c|}
\hline Categoria ou porte & $\begin{array}{c}\text { Taxa de geração } \\
\text { (kg.hóspede- } \\
\text { 1.dia-1) }\end{array}$ & Localização & Fonte \\
\hline $\begin{array}{c}\text { Hotel } 5 \text { estrelas com } 252 \\
\text { quartos (café da manhã, } \\
\text { almoço e jantar (à la } \\
\text { carte) }\end{array}$ & 2,55 & \multirow{2}{*}{$\begin{array}{l}\text { Malta } \\
\text { (Itália) }\end{array}$} & \multirow{2}{*}{$\begin{array}{l}\text { Camilleri-Fenech et al., } \\
2020\end{array}$} \\
\hline $\begin{array}{l}\text { Hotel com } 5 \text { estrelas com } \\
252 \text { quartos (café da } \\
\text { manhã, almoço e jantar } \\
\text { (buffet) }\end{array}$ & 2,87 & & \\
\hline Sem classificação & 1,08 & \multirow{6}{*}{$\begin{array}{l}\text { Kerbala } \\
\text { (Iraque) }\end{array}$} & \multirow{6}{*}{ Abdulredha et al., 2018} \\
\hline 1 estrela & 0,83 & & \\
\hline 2 estrelas & 0,9 & & \\
\hline 3 estrelas & 0,83 & & \\
\hline 4 estrelas & 1,22 & & \\
\hline Média & 0,89 & & \\
\hline Guest House (8 quartos) & 0,6 & \multirow{6}{*}{ Hue (Vietnã) } & \multirow{6}{*}{ Son et al., 2018} \\
\hline 1 estrela (19 quartos) & 0,6 & & \\
\hline 2 estrelas (37 quartos) & 0,48 & & \\
\hline 3 estrelas (72 quartos) & 1,71 & & \\
\hline 4 estrelas (137 quartos) & 2,32 & & \\
\hline 5 estrelas (162 quartos) & 6,57 & & \\
\hline $\begin{array}{c}\text { Pequeno porte (até } 20 \\
\text { quartos) }\end{array}$ & 1,34 & \multirow{4}{*}{$\begin{array}{l}\text { Hoi An } \\
\text { (Vietnã) }\end{array}$} & \multirow{4}{*}{ Phu et al., 2018} \\
\hline $\begin{array}{c}\text { Médio porte }(20 \text { a } 80 \\
\text { quartos) }\end{array}$ & 2,69 & & \\
\hline $\begin{array}{c}\text { Grande porte ( } 80 \text { a } 217 \\
\text { quartos) }\end{array}$ & 6,29 & & \\
\hline Média & 2,28 & & \\
\hline Um Hotel com 74 quartos & 0,32 & Brasil & Guidoni et al., 2018 \\
\hline Guest House (17 leitos) & 0,9 & \multirow{4}{*}{$\begin{array}{l}\text { Pahalgam } \\
\text { (Índia) }\end{array}$} & \multirow{4}{*}{ Bashir e Goswami 2016} \\
\hline Pequeno porte (42 leitos) & 1,7 & & \\
\hline Médio porte (53 leitos) & 1,9 & & \\
\hline Grande porte (114 leitos) & 2,9 & & \\
\hline $\begin{array}{l}\text { Um Hotel com } 118 \\
\text { quartos } *\end{array}$ & 1 & $\begin{array}{c}\text { Kuala Lumpur } \\
\text { (Malásia) }\end{array}$ & $\begin{array}{c}\text { Papargyropoulou, et al., } \\
2016 \\
\end{array}$ \\
\hline Hotel com 74 quartos & 0,38 & Brasil & Peruchinn et al., 2015 \\
\hline $\begin{array}{l}68 \text { hotéis do grupo } \\
\text { Rezidor com uma média } \\
\text { de } 220 \text { quartos** }\end{array}$ & 1,26 & $\begin{array}{c}\text { Europa, Ásia e } \\
\text { África }\end{array}$ & $\begin{array}{l}\text { The Rezidor Group, } \\
2014\end{array}$ \\
\hline $\begin{array}{c}\text { Um Hotel com } 5 \text { estrelas } \\
\text { (média) }\end{array}$ & 1,5 & Egito & Ball e Taleb, 2011 \\
\hline
\end{tabular}

*Resíduos gerados apenas pelo restaurante do hotel; **Taxa de geração média dos 68 hotéis analisados Fonte: Autores.

Estudando a geração de resíduos sólidos em um restaurante de um hotel cinco estrelas localizado em Kuala Lumpur Malásia, Papargyropoulou, et al. (2016) estimam uma taxa de geração per capita de 1 kg.hóspede-1.dia-1. Também investigando 
a geração de resíduos sólidos em um restaurante de um hotel cinco estrelas localizado em Malta, Itália, Camilleri-Fenech et al. (2020) concluem que a geração diária de resíduos sólidos no hotel depende do tipo de refeição que o hóspede faz, ou seja, um hóspede que consome café da manhã, almoço e jantar à la carte gera 2,55 kg/dia, de resíduos sólidos, enquanto que um hóspede que se alimenta no café da manhã, almoço e jantar (buffet) produz 2,87 kg/dia de resíduos sólidos.

Segundo o The Rezidor Hotel Group (2014), os resíduos sólidos de 68 hotéis desta empresa hoteleira têm como origem a preparação dos alimentos, o consumo de bens embalados e em atividades de limpeza. Os hotéis do grupo estão localizados na Europa, Ásia e África, possuindo em média 220 quartos e tendo uma geração per capita média de 1,26 kg.hóspede-1.dia-1 de resíduos sólidos.

Guidoni et al. (2018) e Peruchinn et al. (2015) analisaram a geração de resíduos sólidos de um hotel de 74 quartos, que pode ser classificado como de três estrelas por Son et al. (2018) e de médio porte por Phu et al. (2018). Contudo, a geração per capita de resíduos sólidos deste hotel, localizado no Brasil, foi estimada em 0,32 kg.hóspede-1.dia-1 por Guidoni et al. (2018) e 0,38 kg.hóspede-1.dia-1 por Peruchinn et al. (2015), taxas bem menores em relação aos valores encontrados por Son et al. (2018) e Phu et al. (2018). Isso pode ocorrer devido aos fatores que influenciam na geração de resíduos sólidos no setor hoteleiro, como os serviços ofertados pelo empreendimento, e também pela diferença de cultura entre os países analisados - Vietnã e Brasil (Abdulredha et al., 2018; Phu et al., 2018; Bashir; Goswami, 2016).

Dessa forma, como mostra a tabela 1, há uma variação elevada na taxa de geração de resíduos sólidos oriundos do setor hoteleiro, sendo a taxa mínima identificada igual a 0,32 kg.hóspede-1.dia-1 (Guidoni et al., 2018) e a máxima de 6,57 kg.hóspede1.dia-1 (Son et al., 2018). A Tabela 1 ainda apresenta esta variação com inúmeros parâmetros, como serviços ofertados, número de quartos, classificação e porte do hotel, e número de leitos.

\subsection{Composição dos resíduos sólidos gerados no setor hoteleiro}

Da mesma forma que a taxa de geração per capita, a composição dos resíduos sólidos varia de acordo com as características do hotel, como os hábitos alimentares dos hóspedes, padrão de consumo e serviços oferecidos pelo estabelecimento (Singh; Cranage; Natha, 2014; Zorpas et al., 2014). Agyeiwaah (2020) mostra que os resíduos sólidos gerados em hotéis de pequeno porte da cidade de Cape Coast, Gana, são formados por restos de alimentos, materiais plásticos, borrachas, papéis, e latas de alumínio, além de rejeitos, como o papel higiênico.

Phu et al. (2019), em pesquisa realizada com 120 hotéis da cidade de Hoi An, Vietnã, mostram os resíduos sólidos gerados em hotéis de grande porte são formados por $70 \%$ de resíduos orgânicos e 30\% de resíduos inorgânicos, já para os hotéis de hotéis de pequeno porte, a composição é de 60\% para resíduos orgânicos e 40\% inorgânicos. Anteriormente, Phu et al. (2018) haviam reportado que a composição média dos resíduos sólidos gerados nestes hotéis era de 58,5\% de resíduos orgânicos, 30,6\% de resíduos secos, como papel, plástico, alumínio e vidro, $1 \%$ de resíduos perigosos e 9,9\% de outros tipos de resíduos. Hoang, Fujiwara e Phu (2017) analisando a geração de resíduos sólidos de nove hotéis da cidade Hoi An, reportam que, em média, os resíduos gerados nestes estabelecimentos são formados por 56,2\% de resíduos orgânicos, 33,4\% de resíduos secos, $1 \%$ de resíduos perigosos e 9,3\% de outros tipos de resíduos.

Peruchinn et al. (2015) reportaram que a composição dos resíduos sólidos gerados em um hotel da região Sul do Brasil foi de $49 \%$ de resíduos orgânicos, $27 \%$ de resíduos secos, $14 \%$ de resíduos perigosos e $10 \%$ de outros tipos de resíduos. Em um estudo realizado em hotel de porte semelhante ao que os autores anteriormente citados realizaram sua pesquisa, Guidoni et al. (2018) chegaram a um resultado semelhante: a composição de resíduos foi de $44 \%$ de resíduos orgânicos, 34\% de resíduos secos, 15\% de resíduos perigosos e 7,2\% de outros tipos de resíduos. Analisando a geração de resíduos sólidos em 19 hotéis de Marechal Deodoro, município do Nordeste brasileiro, Dias et al. (2019) mostram que os resíduos orgânicos são os principais responsáveis pelo volume de resíduo gerado nos empreendimentos. Segundo os autores, a presença de restaurantes é um fator primordial para 
que isso ocorra. A Tabela 2 ilustra a composição dos resíduos sólidos gerados no setor hoteleiro.

Tabela 2. Composição dos resíduos sólidos gerados no setor hoteleiro.

\begin{tabular}{|c|c|c|c|c|c|c|}
\hline Fonte & Localização & $\begin{array}{c}\text { Resíduos } \\
\text { Orgânicos } \\
(\%)\end{array}$ & $\begin{array}{c}\text { Resíduos } \\
\text { Secos }(\%)\end{array}$ & $\begin{array}{c}\text { Resíduos } \\
\text { Perigosos } \\
(\%) \\
\end{array}$ & $\begin{array}{c}\text { Resíduos } \\
\text { Diversos } \\
(\%) \\
\end{array}$ & $\begin{array}{c}\text { Potencial de } \\
\text { Reciclagem } \\
(\%)\end{array}$ \\
\hline Speier et al., $2019 *$ & $\begin{array}{l}\text { Mysore } \\
\text { (Índia) }\end{array}$ & 58,8 & 27,9 & 0,0 & 13,3 & 86,7 \\
\hline Phu et al., 2018 & $\begin{array}{l}\text { Hoi An } \\
\text { (Vietnã) }\end{array}$ & 58,5 & 30,6 & 1,0 & 9,9 & 89,1 \\
\hline Guidoni et al., 2018 & Brasil & 43,7 & 34,0 & 15,1 & 7,2 & 77,7 \\
\hline Son et al. 2018 & $\begin{array}{c}\text { Hue } \\
\text { (Vietnã) }\end{array}$ & 54,3 & 30,0 & 0,0 & 15,7 & 84,3 \\
\hline $\begin{array}{l}\text { Chaabane, Nassour } \\
\text { e Nelles, } 2018\end{array}$ & $\begin{array}{l}\text { Hammamet } \\
\text { (Tunísia) }\end{array}$ & 58,0 & 36,0 & 1,0 & 5,0 & 94,0 \\
\hline $\begin{array}{l}\text { Hoang, Fujiwara e } \\
\text { Phu, } 2017\end{array}$ & $\begin{array}{l}\text { Hoi An } \\
\text { (Vietnã) }\end{array}$ & 56,2 & 33,4 & 1,0 & 9,3 & 89,6 \\
\hline $\begin{array}{c}\text { Bashir e Goswami } \\
2016\end{array}$ & $\begin{array}{l}\text { Pahalgan } \\
\text { (Índia) }\end{array}$ & 65,0 & 21,0 & 1,0 & 14,0 & 86,0 \\
\hline $\begin{array}{l}\text { Peruchinn et al., } \\
2015\end{array}$ & Brasil & 49,2 & 26,8 & 13,9 & 10,1 & 76,0 \\
\hline $\begin{array}{l}\text { Appaw-Agbola e } \\
\text { Freeman, } 2015\end{array}$ & Ho (Gana) & 20,3 & 57,6 & 0,0 & 22,0 & 77,9 \\
\hline Zorpas et al., 2014 & Chipre & 42,0 & 31,1 & 2,2 & 24,7 & 73,1 \\
\hline $\begin{array}{l}\text { Singh, Cranage e } \\
\text { Natha, } 2014\end{array}$ & Índia & 59,7 & 39,4 & 0,0 & 0,8 & 99,1 \\
\hline \multirow[t]{2}{*}{ Dangi, et al., 2011} & $\begin{array}{l}\text { Kathmandu } \\
\text { (Nepal) }\end{array}$ & 57,8 & 38,6 & 0,4 & 3,2 & 96,4 \\
\hline & MEDIANA & 57,00 & 32,25 & 1,00 & 10,00 & 86,35 \\
\hline
\end{tabular}

Fonte: Autores.

Son et al. (2018) mostraram que a composição média dos resíduos gerados em 45 hotéis da cidade de Hue, também no Vietnã, foi de 54,3\% de resíduos orgânicos, incluindo desperdício de alimentos e material verde, 30\% de resíduos secos, e 15,7\% de resíduos diversos. Os autores ainda estimam que a participação de resíduos orgânicos é mais elevada em Guest Houses $(57,4 \%)$ e em hotéis cinco estrelas $(56,6 \%)$. Os resíduos secos são representados por recipientes e embalagens de plástico e papéis diversos. Analisando a geração de resíduos sólidos da cidade de Hammamet, Tunísia, Chaabane, Nassour e Nelles (2018) estimam que os hotéis da cidade geraram resíduos sólidos compostos por 58\% de orgânicos, 36\% de resíduos secos, como papel, cartões, embalagens de plástico e de vidro, e copos de plástico, $1 \%$ de resíduos perigosos e $5 \%$ de outros tipos de resíduos.

Segundo Zorpas et al. (2014), a composição média dos resíduos sólidos de hotéis do Chipre era constituída de $42 \%$ de resíduos orgânicos, $31 \%$ de resíduos secos, 2,20\% de resíduos perigosos e 24,7\% de outros tipos de resíduos. De acordo com Singh, Cranage e Natha, (2014), 60\% de resíduos orgânicos e 39\% de resíduos recicláveis compõem os resíduos sólidos gerados em resorts localizados na Índia, como latas de alumínio e aço, papel, embalagens de papelão e plástico. Os autores justificam o 
alto percentual de resíduos orgânicos pelos hábitos alimentares, padrão de consumo e estilo de vida dos indianos. Já Dangi et al. 2011 estimam que a geração média de 271 hotéis da cidade de Kathmandu, Nepal, é de 57,8\% de resíduos orgânicos, 38,6\% de resíduos secos, como metal, vidro e papel, 0,4\% de resíduos perigosos, e 3,2\% de outros tipos de resíduos, como resíduos de construção.

Analisando a geração de resíduos em quatro hotéis da cidade de Ho (Gana), Appaw-Agbola e Freeman (2015) concluem que os resíduos gerados nestes empreendimentos são compostos por 57,65\% de resíduos secos, como vidro, papel e plástico, 20,32\% de resíduos orgânicos, 22,03\% de outros tipos de resíduos, como a borracha. Entre os resíduos secos, o papel é o que é encontrando em mais abundancia (21,43\%). Dos artigos até aqui analisados, este foi o único em que os resíduos orgânicos não são maioria na composição dos resíduos sólidos gerados em hotéis.

Assim, como mostrado na tabela 2, os resíduos orgânicos e os secos constituem grande parte da composição dos resíduos sólidos gerados no setor hoteleiro. Estes resíduos são formados por (mediana dos percentuais reportados na literatura) $57 \%$ de resíduos orgânicos, $32 \%$ por resíduos secos, $1 \%$ de resíduos perigosos e $10 \%$ de outros tipos de resíduos. Assim, percebe-se que o grande potencial de reciclagem que há no setor hoteleiro, já que aproximadamente $86 \%$ dos resíduos gerados em hotéis são passíveis de reciclagem, seja por meio da compostagem, para os resíduos orgânicos, ou reutilização, reciclagem e aproveitamento energético, no caso dos resíduos secos. Alguns autores reportam um potencial maior que a média, como Singh, Cranage e Natha, (2014), com 99,1\%, Dangi, et al. (2011), cujo potencial de reciclagem apresentando é de 96,4\%, e Chaabane, Nassour e Nelles (2018) com 94\% dos resíduos sendo formados por resíduos orgânicos e secos.

\subsection{Destinação dos resíduos gerados em hotéis}

Os resíduos gerados no setor hoteleiro apresentam um grande potencial para reciclagem, já que aproximadamente $86 \%$ são compostos por resíduos orgânicos e secos. Contudo, apesar deste grande potencial, poucos hotéis destinam seus resíduos de forma ambientalmente adequada, como a compostagem ou a coleta seletiva, visando sua reutilização, reciclagem ou aproveitamento energético.

Em pesquisa realizada com 33 hotéis da cidade de Hammamet (Tunísia), Chaabane, Nassour e Nelles (2018) citam que $83 \%$ dos hotéis enviam seus resíduos para o aterro sanitário da cidade, enquanto que apenas $17 \%$ desenvolvem medidas de reutilização e reciclagem. Um cenário semelhante foi descrito por Appaw-Agbola e Freeman (2015) que em pesquisa com quatro hotéis da cidade de Ho (Gana) reportam que todos os hotéis analisados destinam seus resíduos para aterros sanitários, incluindo resíduos orgânicos, alumínios e plásticos, com exceção para o papel e a borracha que são incinerados. Em Pahalgan, cidade da Índia em que as atividades turísticas contribuem com $74 \%$ dos resíduos sólidos gerados, os resíduos sólidos urbanos têm como disposição final um lixão localizado em uma floresta aberta às margens de um córrego de água doce (Bashir \& Goswami, 2016).

Em pesquisa realizada em Cardiff, País de Gales, com nove hotéis de pequeno porte, classificados pelos autores como aqueles que possuem menos de 30 quartos, Radwan, Jones e Minili (2010) descrevem que estes hotéis usam o aterro sanitário como a principal forma de disposição dos resíduos, sem que haja separação entre os resíduos gerados. Os gerentes dos hotéis justificam essa prática pela ausência de espaço físico para a instalação de diferentes coletores, bem como afirmam que o processo de segregação é demorado e caro. Além disso, afirmam que a falta do serviço de coleta seletiva porta-a-porta é o principal dificultador para a implantação de um sistema de reciclagem. Outra justificativa foi dada por um gerente de um hotel de pequeno porte a Filimonau e Tochukwu (2020), ao afirmar que o hotel deve pensar primeiro em como investir e manter sua atividade, e que o meio ambiente não é sua prioridade.

Pesquisas aponta que o porte do hotel tem influência na maior qualidade do gerenciamento dos resíduos, devido a maior disponibilidade de recursos financeiros e humanos disponíveis. Em pesquisa realizada com 18 hotéis do Líbano, nove considerados de grande porte, com mais de 100 quartos, e nove de pequeno porte, com menos de 100 quartos, Ghadban, Shames 
e Mayaleh (2017) concluem que todos os hotéis de grande porte possuíam programas de gerenciamento de resíduos sólidos, ao passo que esses programas foram identificados em apenas três hotéis de pequeno porte. Os autores justificaram esta hipótese coma realização do Teste T Student cujo resultado rejeitou a hipótese nula de que o gerenciamento dos resíduos sólidos é independente de seu porte.

Son et al. (2018) mostram que hotéis de três a cinco estrelas da cidade de Hue (Vietnã) apresentam taxas de segregação de resíduos superiores em relação aos hotéis de uma estrela. Por outro lado, em pesquisa realizada com hotéis de três a cinco estrelas, localizados no Zimbábue (4 hotéis) e na África do Sul (4 hotéis), Mbasera et al. (2016) mostram que apenas um hotel realiza a segregação dos resíduos sólidos na sua fonte de geração, atividade básica para o gerenciamento adequado dos resíduos sólidos.

Abordando a gestão ambiental de 159 hotéis portugueses, classificados entre duas e cinco estrelas, Sousa e Eusébio (2013) mostram que as práticas relacionadas a redução do consumo de energia, água e resíduos, nesta ordem, são as mais executadas pelos hotéis. Em relação à gestão de resíduos sólidos, a coleta de resíduos perigosos, como pilhas e baterias, é a atividade mais comum entre os hotéis analisados, seguido da coleta seletiva e da reutilização de mobilhados.

Analisando 30 hotéis classificados entre três e cinco estrelas e localizados nos Emirados Árabes, Al-Aomar e Hussain (2017) afirmam que as práticas verdes mais comuns adotadas por estes empreendimentos estão relacionadas a redução do consumo de água e energia, seguida pela diminuição na geração de resíduos. Segundo os autores, a reciclagem e a reutilização são práticas adotadas na cadeia de abastecimento do hotel, como por exemplo a reciclagem de embalagens de plástico. Segundo Reyes-Santiago, Sánchez-Medina e Díaz-Pichardo (2017), quando um hotel implementa algum tipo de atividade verde, ele está mais propenso a se envolver em outras atividades de mesma natureza.

Assim, devido ao grande potencial de reciclagem existente, é necessário que os resíduos sólidos gerados no setor hoteleiro sejam geridos de forma eficiente, visando sempre a não geração, redução, reutilização, reciclagem, destinação e disposição final ambientalmente adequada. Com o objetivo de reduzir o montante de resíduos sólidos destinados à aterros sanitários e lixões, é preciso que haja um incentivo por meio de políticas públicas, principalmente para hotéis de pequeno porte, para que estes empreendimentos implantem medidas de minimização, como a separação dos resíduos, coleta seletiva e a compostagem.

Phu et al. (2019) mostram que as práticas de gestão dos resíduos, a promulgação de políticas públicas e a otimização de benefícios econômicos são os principais fatores para o aprimoramento das práticas de reciclagem, com importâncias de 37,75\%, $24,96 \%$ e $15,11 \%$, respectivamente. Em relação às práticas de gestão de resíduos, os autores determinam que a separação dos resíduos é o fator mais importante, com uma percentagem ponderada de 55,8\%, seguida da reciclagem e da compostagem, com $32 \%$ e 12,2\%, respectivamente. Segundo Matter et al. 2013, a separação apropriada dos resíduos é capaz de reduzir o volume gerado de rejeitos, diminuindo, assim, os impactos ambientais negativos causados por eles.

\subsection{Influência das atividades turísticas na geração de resíduos sólidos urbanos}

Os hotéis são grandes geradores de resíduos sólidos, e assim como outras atividades turísticas, destinam seus resíduos para aterros sanitários, afetando diretamente o sistema de gerenciamento de resíduos sólidos urbanos de regiões turísticas. (Bashir \& Goswami, 2016; Appaw-Agbola \& freeman, 2015; Radwan; Jones; Minili, 2010). Um exemplo é relatado por Chaabane, Nassour e Nelles (2018) que mostram que em 2017 os hotéis da cidade de Hammamet, Tunísia, foram responsáveis por gerar aproximadamente 15 toneladas de resíduos sólidos, o que representa 45,5\% do total de resíduos gerados na cidade neste ano. Sealey e Smith (2014) apresentam resultados ainda mais impressionantes, segundo os autores, em 2013 o Sandals Emerald Bay, um resort com 245 quartos, foi responsável por até 35\% do total de resíduos sólidos gerados na Ilha de Exuma, Bahamas, a uma taxa de 4,8 toneladas/dia, sendo $56 \%$ destes resíduos passíveis de reciclagem por meio da compostagem 
Focando em outras atividades turísticas, além dos hotéis, Saito (2013) estima que restaurantes, hotéis, locadoras de automóveis e campos de golfe, são responsáveis por 10,7\% de toda a geração de resíduos sólidos na ilha do Hawai. Em pesquisa visando analisar a geração de resíduos sólidos da área turística da cidade de Pariman (Indonésia), Aziz e Mira (2019) mostram que os shoppings são os empreendimentos turísticos que mais contribuem para a geração de resíduos sólidos nesta região (76\%), seguido dos hotéis $(11,28 \%)$ e restaurantes $(6,77 \%)$.

Alcay, Montañes e Fernández (2020) mostram que regiões da Espanha onde a economia depende primordialmente do turismo, como Ilhas Canárias, Ilhas Beleares e Múrcia, a geração média de resíduos sólidos é o dobro em comparação com outras regiões. Neste contexto, Bashir e Goswami (2016) relatam que para a cidade de Pahalgan (Índia), onde o turismo é a principal fonte de renda, as atividades contribuem fortemente com a geração de resíduos, com os hotéis e restaurantes sendo responsáveis por $74 \%$ do total de resíduos sólidos gerados na cidade. Resultado semelhante foi descrito por Zorpas et al. (2014) que relataram que até 75\% dos resíduos sólidos gerados na cidade de Paralimni, no Chipre, são oriundos de atividades turísticas.

Diversas pesquisas abordam o impacto direto das atividades turísticas no sistema de gerenciamento de resíduos sólidos. Neste sentido, Diaz-Farina, Hernandéz e Fumero- Padrón (2020) mostram que um turista adicional representa o acréscimo de 0,40 kg/dia na geração de resíduos sólidos da cidade de Tenerife, Espanha, divididos em 0,33 kg/dia oriundos do setor de acomodações e $0,07 \mathrm{~kg} /$ dia gerados pelos serviços de comidas e bebidas. Ainda segundo os autores, as atividades turísticas são responsáveis por $11,30 \%$ da geração de resíduos sólidos na cidade.

Para a região espanhola das Ilha Baleares, Estay-Ossandon e Nieto (2018) estimam que um turista adicional, ou seja, uma visitante com estadia média de 10 dias, causa um aumento de $1,78 \mathrm{~kg} /$ dia na geração total de resíduos sólidos urbanos em relação ao ano anterior, enquanto que a população residente é responsável pelo aumento de $1,30 \mathrm{~kg} / \mathrm{dia}$ de resíduos sólidos. Para a Ilha de Minorca, uma das Ilhas Baleares da Espanha, Mateu-Sbert et al. 2013 reportam resultados semelhantes aos dos autores citados anteriormente, estimando que o aumento de $1 \%$ na população turística corresponde ao aumento de $0,28 \%$ na geração de resíduos sólidos durante todo o ano. Os autores ainda consideram que um turista adicional gera 1,31 kg/dia de resíduos, enquanto que um habitante adicional gera $1,48 \mathrm{~kg} / \mathrm{dia}$.

Segundo Oliveira e Turra (2015) durante a alta temporada do turismo no Brasil, a geração média diária de resíduo sólidos aumenta em aproximadamente 30\% em Caraguatatuba e São Sebastião, 20\% em Ilhabela e 90\% em Ubatuba, cidades do Estado de São Paulo. De acordo com Sundrival et al. 2018, a geração média de resíduos sólidos urbanos em Mussoorie, cidade da Índia, é estimada em 18 toneladas/dia, enquanto que a geração a alta temporada do turismo é de 23 toneladas/dia, representando um aumento de $27 \%$.

Corroborando os autores supracitados, Iuras et al., 2020 a fim de determinar a influência das atividades turísticas na geração de resíduos gerados na região de Ivano-Frankivsk (Ucrânia) mostraram que o número de turistas, produto interno bruto e a população local são parâmetros que influenciam em até $84 \%$ a geração de resíduos sólidos da cidade. A partir de um modelo de regressão, os autores obtiveram um coeficiente múltiplo $R=0,92$ e $R^{2}=0,84$.

Assim, pode-se dizer que as atividades turísticas influenciam diretamente no sistema de gerenciamento de resíduos sólidos, seja por meio do aumento na geração de resíduos sólidos ou de sua disposição em aterros sanitários. Além destes fatores, as atividades turísticas também podem ser responsáveis pelo aumento nos custos da coleta dos resíduos sólidos, como mostram Greco, Cenciarelli e Allegrini (2018). Segundo os autores o aumento de 1\% nas dormidas em hotéis italianos aumenta o custo da coleta de papel em $0,26 \%$, com o modelo apresentando $\mathrm{R}^{2}$ igual a 0,58 . Já para o metal, vidro e plástico, este aumento é de $0,14 \%$, com o modelo apresentando $\mathrm{R}^{2}$ igual a 0,80 .

A influência das atividades turísticas sobre a geração de resíduos sólidos urbanos pode ser utilizada como argumento para a cobrança de uma taxa de coleta diferencial imposta aos estabelecimentos comerciais, como os hotéis, em relação às residências. Greco, Cenciarelli e Allegrini (2018) sugerem a cobrança desta taxa em forma de imposto nas diárias dos hotéis, ou 
por taxas adicionais para atividades turísticas, como bares, restaurantes e hotéis. Na Tunísia, por exemplo Chaabane, Nassour e Nelles (2018) reportam que os hotéis pagam um importo referente à $2 \%$ de seu faturamento anual, sendo $1 \%$ destinado para o município, valor utilizado como auxílio nos custos das atividades de coleta de resíduos sólidos e limpeza urbana, e 1\% para o fundo de proteção de destinos turísticos, financiando atividades relacionadas a segurança dos turistas, por exemplo.

\section{Conclusão}

De acordo com os resultados encontrados, o setor hoteleiro se apresenta como um grande gerador de resíduos sólidos, com taxas de geração que variam de 0,32 a 6,57 kg.hóspede-1.dia-1. Os resíduos gerados no setor hoteleiro são, em sua maioria, de origem orgânica (57\%) e secos, como papel, plástico, vidro e alumínio (32\%), mostrando alto potencial para o desenvolvimento de atividades de compostagem e reciclagem. O nível de aproveitamento dos resíduos sólidos gerados em hotéis ainda é baixo, sendo boa parte ainda encaminhada para aterros sanitários. Sendo assim, é de fundamental importância que os resíduos sólidos gerados no setor hoteleiro sejam geridos de forma adequada, visando sempre a sua não geração, redução, reutilização e reciclagem. Devido às características variadas dos empreendimentos hoteleiros, a caracterização e quantificação dos resíduos sólidos gerados por eles gerados, possuem papel fundamental em sua gestão. Através destas é possível identificar qual o tipo de resíduo necessita de mais medidas de minimização, e aperfeiçoando seu sistema de gestão.

Recomenda-se que as pesquisas envolvendo o gerenciamento dos resíduos sólidos gerados no setor hoteleiro se aprofundem nas características do empreendimento, bem como nas particularidades da cultura que o hotel está envolvido. Dessa forma, poderão ser elaborados diagnósticos bem fundamentados sobre a geração de resíduos nestes estabelecimentos.

\section{Referências}

Abdulredha, M., Al khaddar, R., Jordan, D., Kot, P., Abdulridha, A. \& Hashim, K. (2018). Estimating solid waste generation by hospitality industry during major festivals: A quantification model based on multiple regression. Waste Management, 77 (1), 388-400. https://doi.org/10.1016/j.wasman.2018.04.025

Agyeiwaah, E. (2020). The contribution of small accommodation enterprises to sustainable solid waste management. Journal of Hospitality and Tourism Management, 44 (1), 1-9. https://doi.org/10.1016/j.jhtm.2020.04.013.

Al-Aomar. R., \& Hussain, M. (2017). An assessment of green practices in a hotel supply chain: A study of UAE hotel. Journal of Hospitality and Tourism Management, 32 (1), 71-81. https://doi.org/10.1016/j.jhtm.2017.04.002.

Alcay, A., Montañes, A., \& Fernández, M. B. S. (2020). Waste generation in Spain. Do Spanish regions exhibit a similarbehavior?. Waste Management, 112 (1), 66-73. https://doi.org/10.1016/j.wasman.2020.05.029.

Appaw-Agbola, E., \& Freeman, B. (2015). Solid Waste Management in the Tourism Sector of Ghana. A Study of Selected Hotels in Ho the Regional Capital of Volta Region. Journal of Tourism, Hospitality and Sports, 10 (1). https://www.iiste.org/Journals/index.php/JTHS/article/view/24078.

Arbulú, I., Lozano, J., \& Maquieira, R. J. (2015). Tourism and solid waste generation in Europe: A panel data assessment of the Environmental Kuznets Curve. Waste Management, 46 (1), 628-636. https://doi.org/10.1016/j.wasman.2015.04.014.

Aziz, R., \& Mira. (2019). Study of recycling potential of solid waste of tourist area in Pariaman City. IOP Conference Series: Materials Science and Engineering. 602 (1). 10.1088/1757-899X/602/1/012059.

Ball, S., \& Taleb, M. A. (2011). Benchmarking waste disposal in the Egyptian hotel industry. Tourism and Hospitality Research, 11 (1), 1-18. https://doi.org/10.1057/thr.2010.16.

Bashir, S., \& Goswami, S. (2016). Tourism induced Challenges in Municipal Solid Waste Managemen in Hill Towns: Case of Pahalgam. Procedia Environmental Sciences, v. 35 (1), 75 - 89. https://doi.org/10.1016/j.proenv.2016.07.048.

Camilleri-Fenech, M., Sola, H. O., Farreny, R., \& Durany, X. G. (2020). A snapshot of solid waste generation in the hospitality industry. The case of a five-star hotel on the island of Malta. Sustainable Production and Consumption,21 (1), 104-119. https://doi.org/10.1016/j.spc.2019.11.003.

Chaabane, W., Nassour, A., \& Nelles, M. (2018). Solid Waste Management Key Indicator Development for Hotels: A Tunisian Case Study Analysis. Recycling, 3 (4). https://doi.org/10.3390/recycling3040056.

Dangi, M. B., Pretz, C. R., \& Urynowicz, M. A. Gerow, K. G. Reddy, J. M. (2011). Municipal solid waste generation in Kathmandu, Nepal. Journal of Environmental Management, 92 (1), 240-249. https://doi.org/10.1016/j.jenvman.2010.09.005.

Dias, S. K. B. M., Fiori, A. P. S. M., Nascimento, X. V., Mello, G. S. V., \& Miranda, P. R. B. (2019). Percepción ambiental de los gestores de medios de hospedaje El caso de Praia do Francês en el municipio de Marechal Deodoro - Brasil. Estudios y Perspectivas en Turismo, 28 (1), 923 -241. https://www.redalyc.org/jatsRepo/1807/180762638004/html/index.html. 
Diaz-Farina, E., Hernández, J. D., \& Fumero- Padrón, N. (2020). The contribution of tourism to municipal solid waste generation: A mixed demand-supply approach on the island of Tenerife. Waste Management, 102 (1), 587-597. https://doi.org/10.1016/j.wasman.2019.11.023.

Estay-Ossandon, C., \& Nieto, A. M. (2018). Modelling the driving forces of the municipal solid waste generation in touristic islands. A case study of the Balearic Islands (2000-2030). Waste Management, 75 (1), 70-81. https://doi.org/10.1016/j.wasman.2017.12.029.

Filimonau, V., \& Tochukwu, C. O. (2020). Exploring managerial approaches to mitigating solid waste in hotels of Lagos, Nigeria. Journal of Cleaner Production, 270. https://doi.org/10.1016/j.jclepro.2020.122410.

Ghadban, S., Shames, M., \& Mayaleh, H. A. (2017). Trash Crisis and Solid Waste Management in Lebanon-Analyzing Hotels' Commitment and Guests' Preferences. Journal of Tourism Research Hospitality, SciTechnol, 6 (3). 10.4172/2324-8807.1000171.

Gil, A. C. (2002). Como elaborar projetos de pesquisa. (4a ed.): Atlas.

Greco, G., Cenciarelli, V. G., \& Allegrini, M. (2018). Tourism's impacts on the costs of municipal solid waste collection: Evidence from Italy. Journal of Cleaner Production, 177, 62-68. https://doi.org/10.1016/j.jclepro.2017.12.179.

Guidoni, L. L. C., Peruchini, B., Corrêa, 1. B., Marques, R. V., Vieira, 1. A., Siqueira, T. M., \& Corrêa, E. A. (2018). Solid waste generation in a hotel event service. Rev. Int. Contam. Ambiente, 34 (2), 237-247. http://dx.doi.org/10.20937/rica.2018.34.02.05.

Hoang, M. G., Fujiwara, T., \& Phu, S. T. P. (2017). Municipal waste generation and composition in a tourist city - Hoi An, Vietnam. Journal of JSCE, 5, 123132. 10.2208/journalofjsce.5.1_123.

IBGE. (2017). Pesquisa de Serviços de Hospedagem: 2016. Instituto brasileiro de geografia e estatística https://biblioteca.ibge.gov.br/visualizacao/livros/liv100623.pdf".

Iuras, I., Raiter, P., Korobeinykova, Y., \& Poberezhna, l. (2020). Methodology of actors analysis and modeling of the amounts of solid municipal waste generation within tourist destinations. Ecological Questions, 31 (2), 63-69. http://dx.doi.org/10.12775/EQ.2020.014.

Mateu-Sbert, J., Ricci-Cabello, I., Villalonga-Olives, E., \& Cabeza-Irigoyen, E. (2013). The impact of tourism on municipal solid waste generation: the case of Menorca Island (Spain). Waste Manag. 33 (12), 2589-2593. https://doi.org/10.1016/j.wasman.2013.08.007.

Matter, A., Dietschi, M., \& Zurbrügg, C. (2013). Improving the informal recycling sector through segregation of waste in the household - The case of Dhaka Bangladesh. Habitat International, 38, 150 -156. https://doi.org/10.1016/j.habitatint.2012.06.001.

Mbasera, M., Plessis, D., Saayman, M., \& Krugerm, M. (2016). Environmentally-friendly practices in hotel. Acta Commercii - Independent Research Journal in the Management Sciences, 16 (1). https://doi.org/10.4102/ac.v16i1.362.

Munõz, E., \& Navia, R. (2015). Waste management in touristic regions. Waste Management \& Research, 33 (7), 593-594. https://doi.org/10.1177/0734242X15594982.

Oliveira, A. L., \& Turra, A. (2015). Solid waste management in coastal cities: where are the gaps? Case study of the North Coast of São Paulo, Brazil. Revista de Gestão Costeira Integrada / Journal of Integrated Coastal Zone Management, 15 (4), 453-465. 10.5894/rgci544.

Papargyropoulou, E., Wright, N., Lozano, R., Steinberger, J., Padfield, R., \& Ujang, Z. (2016). Conceptual framework for the study of food waste generation and prevention in the hospitality sector. Waste Management, 49, 326-336. https://doi.org/10.1016/j.wasman.2016.01.017.

Pereira A. S., et al. (2018). Metodologia da pesquisa científica. Ed. https://repositorio.ufsm.br/bitstream/handle/1/15824/Lic_Computacao_Metodologia-Pesquisa-Cientifica.pdf?sequence=1 .

Peruchinn, B., Ferrão, A. L. L. C., Guidoni, L. L. C., Corrêa, E. K., \& Corrêa, L. B. (2015). Estudo da geração dos resíduos sólidos em hotel. Revista Turismo Visão e Ação - Eletrônica, 17 (2), 301-322. https://doi.org/10.14210/rtva.v17n2.p301-322.

Phu, S. T. P., Fujiwara, T., Hogang, M. G., Pham, V. D., \& Than, M. T. (2019). Waste separation at source and recycling potential of the hotel industry in Hoi An city, Vietnam. Journal of Material Cycles and Waste Management, 21, 23-34. https://link.springer.com/article/10.1007/s10163-018-0807-5.

Phu, S.T. P., Hoang, M.G., \& Fujiwara, T. (2018). Analyzing solid waste management practices for the hotel industry. Global J. Environ. Sci. Manage, 4 (1), 19-30. 10.22034/GJESM.2018.04.01.003.

Pirani, S. I., \& Arafat, H. A. (2014). Solid waste management in the hospitality industry: a review. J. Environ. Manage, 146, 320-336. https://doi.org/10.1016/j.jenvman.2014.07.038.

Radwan, H. R. I., Jones, E., \& Minili, D. M. I. (2010)). Managing solid waste in small hotel. Journal of Sustainable Tourism, 18 (2), 175-190. https://doi.org/10.1080/09669580903373946.

Reyes-Santiago, M. R., Sánchez-Medina, P. S., \& Díaz-Pichardo, R. (2017). Eco-innovation and organizational culture in the hotel industry. International Journal of Hospitality Management, 65, 71-80. https://doi.org/10.1016/j.ijhm.2017.06.001.

Saito, O. (2013). Resource Use and Waste Generation by the Tourism Industry on the Big Island of Hawaii. Journal of Industrial Ecology, v. x. https://doi.org/10.1111/jiec.12007.

Sealey, k. S., \& Smith, J. (2014). Recycling for small island tourism developments: Food waste composting at Sandals Emerald Bay, Exuma, Bahamas. Resources, Conservation and Recycling. 92, 25-37. https://doi.org/10.1016/j.resconrec.2014.08.008.

Singh, N., Cranage, D. A., \& Natha, A (2014). Estimation of GHG Emission from Hotel Industry. Anatolia - An International Journal of Tourism and Hospitality Research, 25, 39-48. https://doi.org/10.1080/13032917.2013.822817. 
Research, Society and Development, v. 10, n. 1, e16810111635, 2021 (CC BY 4.0) | ISSN 2525-3409 | DOI: http://dx.doi.org/10.33448/rsd-v10i1.11635

Son, 1. H., Matsui, Y., Trang, T. T., \& Than, N. P. (2018). Estimation of the Solid Waste Generation and Recycling Potential of the Hotel Sector: A Case Study in Hue City, Vietnam. Journal of Environmental Protection, 9, 751-769. https://doi.org/10.4236/jep.2018.97047.

Sousa, B., \& Eusébio, C. A. (2013) Análise da gestão ambiental nos hotéis portugueses. Revista portuguesa e brasileira de gestão, 12, (21). http://bibliotecadigital.fgv.br/ojs/index.php/rbpg/article/view/21298.

Subbiah, K., \& Kannan, S. (2011). The Eco-friendly Management of Hotel Industry. In: International Conference on Green Technology and Environmental Conservation - GTEC. https://ieeexplore.ieee.org/document/6167681.

Sundrival, S., Shridhar, V., Madhwal, S., Pandy, K., \& Sharma, V. (2018). Impacts of tourism development on the physical environment of Mussoorie, a hill station in the lower Himalayan range of India. J. Mt. Sci, 15 (10), 2276 - 2291. https://doi.org/10.1007/s11629-017-4786-0.

The Rezidor Hotel Group. 2014. Sustainability Report: Creating Value through Responsible Business. https://www.radissonhospitalityab.com/staticfiles/d634029f-1143-464a-bf7e-d0ff0d15a2d3.

WTTC. (2018). Travel \& Tourism: Global economic impact \& issues 2018. World travel \& tourism council. London, United Kingdom. https://wttc.org/Research/Economic-Impact.

WTTC. (2017). Travel \& Tourism. Economic impact 2017 world. World travel \& tourism council. London, United Kingdom: https://wttc.org/Research/Economic-Impact.

Zorpas, A. A., Voukkali, I., \& Loizia, P. (2014). The impact of tourist sector in the waste management plans. Desalination and Water Treatment, 1 (9). https://doi.org/10.1080/19443994.2014.934721. 